\title{
Linfoma no Hodgkin pulmonar primario anaplásico de células grandes Ki-1 positivo. Estudio de un caso y revisión de la literatura
}

\author{
S. BELTRÁN BELTRÁN, Mª.E. DE TOMÁS LABAT*, P. FERRERAS FERNÁNDEZ \\ Servicio de Medicina Interna Complejo Hospitalario de Albacete. *Servicio de Medicina \\ Interna Hospital de Hellín. Albacete
}

\begin{abstract}
PRIMARY PULMONARY KI-1 POSITIVE ANAPLASTIC LARGE CELLS NON-HODGKIN'S. LYMPHOMA. STUDY OF A CASE AND REVIEW OF THE LITERATURE
\end{abstract}

\begin{abstract}
RESUMEN
Los linfomas no Hodgkin anaplásicos de células grandes Ki-l positivo son una entidad recientemente reconocida, poco frecuentes y caracterizados por la expresión del antígeno CD30. Su forma mas frecuente de presentación es la de adenopatías periféricas con afectación mediastínica, acompañada en aproximadamente la mitad de los casos de afectación extranodal, siendo la piel el lugar mas común; y siendo infrecuente que se presenten con afectación del pulmón, la médula ósea y el sistema nervioso central. Por tanto los linfomas no Hodgkin pulmonares primarios anaplásicos de células grandes Ki-l (+) son una entidad clínica rara. Se clasifican y clínicamente se comportan como linfomas de alto grado de malignidad, que en la mayoría de casos se encuentran en estadios clínicos avanzados en el momento del diagnóstico. Se presentan con mayor incidencia en gente joven, donde el pronóstico es mas favorable. Se describen las características clínicas, morfológicas e inmunofenotípicas de este tipo de linfomas.
\end{abstract}

PALABRAS CLAVE: Linfoma no Hodgkin anaplásico de células grandes Ki-l positivo. Linfoma pulmonar primario.

\begin{abstract}
Ki-l positive anaplastic large cell non-Hodgkin's lymphomas are a recently recognized entity, unusual and characterized by the expression of CD30 antigen.The most usual clinical feature is peripheral lymphade nopathy with mediastinal sparing and extranodal disease which occurs in approximately half of the cases, with the skin as the most common site; lung, bone marrow and central nervous system involvement are uncom mon. Therefore primary pulmonary Ki-l positive anaplastic large cell non-Hodgkin's lymphomas are an uncommon clinical entity. Their clas sification and clinical behaviour look like high grade malignancy lymp homas, that in the most cases are presented in advanced stages disease to the diagnostic. They are presented with higher incidence in young people, where prognostic is more favourable. Clinical, morphologic and inmunophenotypic features of this lymphoma type are reported.
\end{abstract}

KEY WORDS: Ki-1 positive anaplastic large cell non-Hodgkin's lymphoma. Primary pulmonary lymphoma.

Beltrán Beltrán S, De Tomás Labat Ma E, Ferreras Fernández P. Linfoma no Hodgkin pulmonar primario anaplásico de células grandes Ki-1 positivo. Estudio de un caso y revisión de la literatura. An Med Interna (Madrid) 2001; 18: 587-590.

\section{INTRODUCCIÓN}

El linfoma pulmonar (LP) se engloba en el contexto de las enfermedades pulmonares linfoproliferativas malignas (1), debiendo situarse el origen de la mayoría de ellos en el BALT, el cual formaría parte del MALT $(2,3,5)$. El concepto de LP primario (LPP) es arbitrario, existiendo varios criterios para su definición $(6,10)$, aunque en la actualidad el criterio consensuado por la mayoría de autores requiere demostrar la ausencia de afectación extraparenquimatosa en el momento del diagnóstico o en un periodo posterior de tres meses, no existiendo acuerdo sobre si la afectación de ganglios linfáticos mediastínicos o la pleura invalida el diagnóstico $(2,4,6)$. El anticuerpo monoclonal (AcM) Ki-1 descrito en 1982 (11), fué relacionado en 1985 por Stein y cols. (12) con neoplasias de células grandes, creando una nueva categoría de linfomas no Hodgkin (LNH) de alto grado de malignidad, que se incluyeron en el grupo de los LNH anaplásicos de células grandes (LACG). El AcM Ki-1 reconoce un antígeno de superficie específico de la célula linfoide maligna llamado antígeno CD30, cuya expresión tiene lugar trás su activación inmunológica y marca un grupo heterogéneo de procesos linfoproliferativos malignos $(11,19)$.

Presentamos el caso de una paciente joven, diagnosticada de LNH pulmonar primario anaplásico de células grandes (LPPACG) Ki-l (+) y que evolucionó favorablemente trás recibir tratamiento con quimioterapia (QT).

Trabajo aceptado: 15 defebrero de 1999

Correspondencia: Silvestre Beltrán Beltrán. C/ de la Informática, 43. 02006 Albacete. 


\section{CASO APORTADO}

Mujer de 18 años de edad sin antecedentes de interés, que ingresó en nuestro Centro por infiltrado en el lóbulo pulmonar superior izquierdo (LSI) de 4 meses de evolución sin respuesta a diversos tratamientos antibióticos. Refería desde hacía 4 meses, tos con expectoración hemoptoica ocasional, astenia, anorexia, perdida de $4 \mathrm{~kg}$ de peso y molestias retroesternales inespecíficas en el último mes. En la auscultación pulmonar presentaba escasos estertores crepitantes en hemicampo superior izquierdo; con resto de EF normal. Las EC mostraban una analítica general, que incluía hemograma, coagulación, VSG, sistemático de orina, autoanalizador con LDH y beta2-microglobulina, proteinograma, inmunoglobulinas, complemento y poblaciones linfocitarias, normal; mantoux anérgico en dos ocasiones; serología a brucella, clamydia, M. pneumoniae, C. burnetti y VIH 1 y 2 negativas; BK esputo en diversas muestras negativas; ECG normal; radiología torácica: condensación pulmonar en LSI de bordes irregulares, cavitada y con brocograma aéreo (Fig. 1); fibrobroncoscopia: sin hallazgos; TAC toraco-abdominal: imagen pulmonar de opacidad nodular, cavitada, de $5 \mathrm{~cm}$ en LSI, con resto normal (Fig. 2); aspirado y biopsia de médula ósea resultaron negativos; y citologias en esputo, BAS, BAL y PAAF transtorácica de la masa pulmonar en LSI: positivas para células tumorales malignas y sospechosas de proceso linfoide. Con la sospecha de LPP y ante la ausencia de tejido para el diagnostico definitivo, se remitió a la paciente a un Hospital

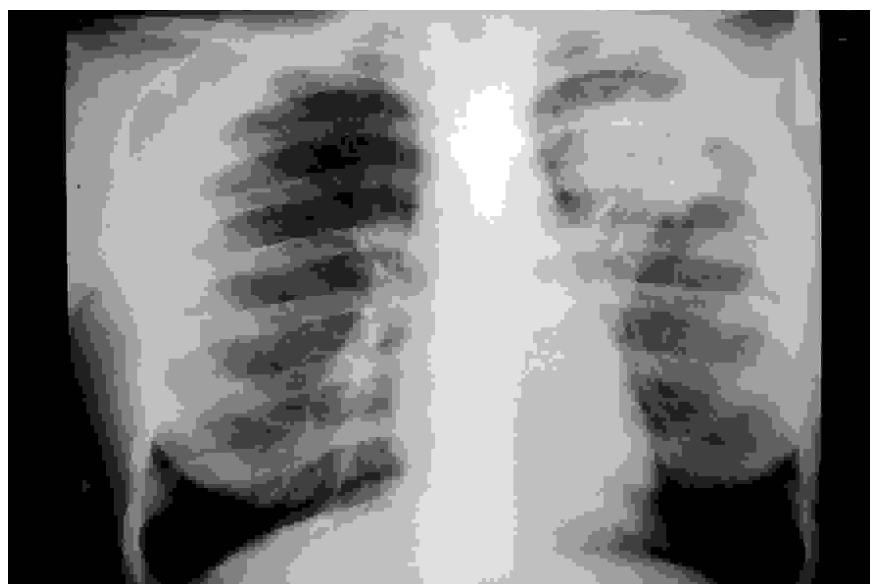

Fig. 1. Radiografía postero-anterior de tórax: imagen de condensación pulmonar a nivel del lóbulo superior izquierdo de bordes irregulares, cavitada y con presencia de broncograma aéreo.

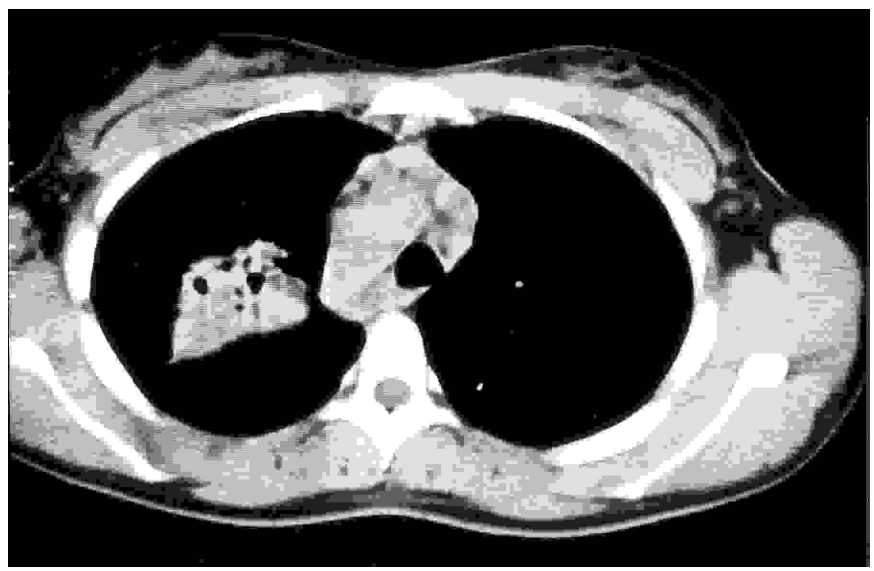

Fig. 2. TAC torácico: imagen pulmonar a nivel del lóbulo superior izquierdo de opacidad nodular, cavitada y con diametro máximo de $5 \mathrm{~cm}$. de referencia para la realización de una biopsia pulmonar, siendo el informe anatomopatológico definitivo de LNH pulmonar anaplásico de células grandes y alto grado $\mathrm{Ki}-1$ (+), mostrando la citología tumoral células grandes con núcleo irregular, nucleolo llamativo y amplio citoplasma claro, existiendo junto al crecimiento tumoral, abundante componente histiocitario con fibrosis intersticial y proliferación vascular; e inmunofenotipo CD30, EMA, CD25, Ki-67 y PCl0 positivos, con CD45 positivo solo en algunas células, marcadores B y T dudosos y CD15 negativo; siendo remitida de nuevo a nuestro Centro para tratamiento. Mes y medio trás su ingreso y previo al inicio del tratamiento quimioterápico, se realizó una RMN torácica que mostró una masa pulmonar en LSI con importante broncograma aéreo y cavitación central, extendiéndose hasta mediastino y contactando con la pleura posterior, pero sin observarse invasión pleural, mediastínica, vascular ni de la grasa subpleural; iniciando posteriormente tratamiento con QT tipo CHOP, recibiendo un total de 8 ciclos con buena tolerancia y respuesta; con gammagrafía ósea normal y mínima imagen de lesión residual en LSI en la radiología y TAC torácicos realizados trás haber finalizado la QT. Actualmente y mas de tres años trás haber finalizado el tratamiento, la paciente esta asintomática e incorporada a su actividad habitual.

\section{DISCUSIÓN}

El LPP es una entidad rara y su frecuencia es menos del $1 \%$ de todos los linfomas; siendo la mayoría LNH de bajo grado de malignidad de estirpe B (2). El LACG Ki-1 (+) es una entidad recientemente reconocida, que se caracteriza por células grandes, pleomórficas, con un patrón de crecimiento sinusal y expresión del antígeno CD30, poco frecuente y con una incidencia del $2-7 \%$ de los LNH $(20,21)$; que para la mayoría de autores presenta predominio en los hombres y mayor incidencia en gente joven, siendo el $70 \%$ menores de 30 años, donde el pronóstico es mas favorable $(14,16,20,25)$. El fenotipo mas frecuente en los LACG Ki-1 (+) es el T en un $60-75 \%$ de casos $(14,20,26)$; siendo su forma mas frecuente de presentación la de adenopatías periféricas con afectación mediastínica, acompañada en un 40-65\% de casos de afectación extranodal, con la piel como lugar mas común, e infrecuente que se presente con afectación del pulmón, la médula ósea y el sistema nervioso central $(13,21,23,24,26)$. Por tanto el LPPACG Ki-l (+) es una entidad clínica rara y de ahí el interés por su presentación. Su etiología es desconocida y se ha asociado a determinadas alteraciones citogenéticas, como una translocación específica que afecta a los cromosomas 2 y 5 t(2;5) (p23;q35) (14,26,29). Los LACG Ki-l (+) se clasifican como LNH de alto grado de malignidad $(30,31)$ y en cerca del $70 \%$ de casos se encuentran en estadíos clínicos III y IV al diagnóstico $(13,26)$; a diferencia de nuestro caso que se encontraba en un estadío IIE. El tiempo de evolución clínica en el momento del diagnóstico suele ser corto, de 3 meses o menos en el $75 \%$ de casos (13), estando generalmente todos los pacientes sintomáticos al diagnóstico, como ocurrió en nuestro caso, y siendo las manifestaciones clínicas tos, hemoptisis, disnea, dolor torácico, auscultandose estertores crepitantes a nivel de la región pulmonar afecta hasta en un $20 \%$ de casos, astenia, anorexia, pérdida de peso, la cual se presenta casi de forma sistemática, y síntomas B $(1,3,13)$. Las alteraciones analíticas en el LPPACG Ki-l (+) son similares a las de otros LNH de alto grado de malignidad; siendo las mas características la elevación de la LDH, beta2-microglobulina e IL-2R, las cuales tienen valor pronóstico y utilidad en el seguimiento posterior, y en el caso de la LDH tanto 
para valorar la respuesta al tratamiento como para detectar una eventual recaída $(5,13,33)$; aunque en los LACG Ki-1 (+) los valores de LDH son irrelevantes para el curso de la enfermedad y no representan un factor desfavorable como en el resto de LNH de alto grado de malignidad (14,26,32). Sin embargo nuestra paciente presentaba una LDH y beta2microglobulina normales. Como en el caso descrito, las técnicas de radiología torácica muestran como forma de presentación mas frecuente del LPPACG Ki-l (+), la de masas o áreas de consolidación parenquimatosa de bordes irregulares y con frecuencia con broncograma aéreo $(3,4,34,35)$, siendo rara la cavitación (36); infrecuente que produzca atelectasias y afectación pleural y mediastínica (3); y muy raro que se manifiesten como lesiones endobroquiales únicas o múltiples $(1,48)$; apareciendo adenopatías hiliares hasta en un $20 \%$ de casos $(1,2)$. En un $20-50 \%$, la fibrobroncoscopia es macroscópicamente normal (3). El diagnóstico definitivo de LPPACG Ki-l (+) se realiza por técnicas de histopatología e inmunohistoquímica, requiriendo casi siempre una biopsia pulmonar abierta $(1,3)$. La mayoría de autores acepta la existencia de dos subtipos histológicos de LACG Ki-1 (+), tipo I y II, propuestos por Chan y cols. (22) en 1989, correspondiendo nuestro caso con el subtipo I; no habiendo sido hallada actualmente una correlación significativa entre estos subtipos histológicos y la evolución clínica y el pronóstico de los mismos $(14,21)$. Hoy en día el estudio inmunofenotípico de los linfomas se considera fundamental, por tanto el diagnóstico de LACG Ki-l (+) ha de sustentarse, además de en la morfología, en el inmunofenotipo, siendo imprescindible que el CD30 sea positivo y presentando con frecuencia el EMA, CD25, CD71, Ki-67, CD45 y HLA-DR positivos (12-14, 20,22,26,39,40). El CD15, propio de la Enfermedad de Hodgkin $(\mathrm{EH})$, suele ser negativo, aunque hasta en un $20 \%$ de casos puede ser positivo $(14,41)$. El análisis genotípico muestra un reordenamiento genético para los receptores beta y gamma de células T (TcR) y más raramente para inmunoglobulinas $(20,39,40)$. Algunos estudios sugieren que en los
LACG Ki-l (+) hay solo dos parámetros clínicos que tienen significación pronóstica: la extensión de la enfermedad y la presencia de síntomas sistémicos (14). La supervivencia de los LPP de alto grado de malignidad es del $60 \%$ a los 5 años y el $75 \%$ presenta una recidiva en un intervalo medio de 33 meses (1). Los LACG Ki-l (+) presentan una buena respuesta a la QT, la cual es similar a la de otros linfomas de alto grado de malignidad $(14,20,26)$; siendo el estadío un factor altamente predictivo para conseguir una remisión completa mantenida (26). Entre los distintos fenotipos de LACG Ki--1 (+) no hay diferencias significativas en el número de remisiones completas, evolución clínica y supervivencia media, la cual es del 50-55\% a los 5 años $(13,14,20)$. Por tanto los LACG $\mathrm{Ki}-\mathrm{l}(+)$ presentan un buen pronóstico y supervivencia respecto a otros tipos de LCG, probablemente por su buena respuesta a la QT $(20,22,26)$. Todavía no hay información suficiente sobre el significado pronóstico de la afectación de sitios menos comunes, como el pulmón y la médula ósea, en los LACG Ki-l (+) (21). Las características histológicas, inmunohistoquímícas y el comportamiento clínico sugieren que existe una íntima relación entre el LACG Ki-1 (+) y la EH $(12,14,20,22,31,39,41)$. Hoy en día, en la mayoría de casos de LNH, la actitud terapeútica debe tener como objetivo la búsqueda de la curación, la cual dependerá fundamentalmente del tipo histológico y en menor grado de su extensión. El tratamiento de elección del LPPACG Ki-l (+) es la QT complementada o no con cirugía y/o RT $(13,14,20,26)$, existiendo todavía controversia en el tipo de QT a emplear $(14,16,44)$, siendo la tendencia actual mas generalizada la de emplear protocolos alternantes, en los cuales se combinan protocolos de primera generación con otros mas agresivos, para así reducir la toxicidad y mejorar la tolerancia de los de tercera generación. Algunos autores proponen que la inmunotoxina Ber-H2-So6, la cual se ha mostrado efectiva en casos de EH refractaria, debería ser empleada para erradicar la enfermedad residual mínima que pudiera haber escapado a la QT convencional en los LACG Ki-l $(14,45,46)$.

\section{Bibliografía}

1. Thompson GP, Utz JP, Rosenow EC, Myers JL, Swensen SJ. Pulmonary lymphoproliferative disorders. Mayo Clin Proc 1993; 68: 804-17.

2. Menárguez J, Carrión JR. Linfomas pulmonares. Med Clin 1995; 105 : 301-2.

3. Cordier JF, Chaílleaux E, Lauque D, Reynaud-Gaubert M, DietemannMolard A, Dalphin JC, et al. Primary pulmonary lymphomas: a clinical study of 70 cases in non inmunocompromised patients. Chest 1993; 103: 201-8.

4. Romero S, González T, Cabezas A, Serralta J, Hernández L, De Juan F. Linfoma pulmonar primitivo. Rev Clin Esp 1985; 177: 90-2.

5. Hernández García MT, Hernández Nieto L. Linfomas no Hodgkin. Medicine 1992; 6(14): 624-38.

6. Papaioannou AN, Watson WL. Primary lymphoma of the lung: an appraisal of its natural history and a comparison with other localizad lymphomas. J Thorac Cardiovasc Surg 1965; 49: 373-87.

7. Colby TV, Carrington CB. Lymphoreticular tumors and infiltrates of the lung. Pathol Ann 1983; 18 (Part 1): 27-70.

8. Kern WH, Crepeau AG, Jones JC. Primary Hodgkin's disease of the lung: report of 4 cases and review of the literature. Cancer 1961; 14: 1151-65.

9. Saltzstein SL. Pulmonary malignant lymphomas and pseudolymphomas: classification, therapy and prognosis. Cancer 1963; 16: 928-55.
10. L'Hoste RJ Jr, Filippa DA, Lieberman PH, Bretsky S. Primary pulmonary lymphomas: a clinicopathologic analysis of 36 cases. Cancer 1984; 54: 1397-406.

11. Schwab U, Stein H, Gerdes J, Lemke H, Kirchner H, Schaadt M, et al. Production of a monoclonal antibody specific for Hodgkin and Sternberg-Reed cells of Hodgkin's disease and a subset of normal lymphoid cells. Nature 1982; 299: 65-7.

12. Stein H, Mason DY, Gerdes J, O’Connor N, Wainscoat J, Pallesen G, et al. The expression of the Hodgkin's disease associated antigen Ki-1 in reactive and neoplastic lymphoid tissue: evidence that Reed-Sternberg cells and histiocytic malignancies are derived from activated lymphoid cells. Blood 1985; 66: 848-58.

13. Rivas C, Obeso G, Piris M, Castrillo JM, Bellas C, Acevedo A, et al. Linfomas no Hodgkin Ki-1: estudio multihospitalario de 21 casos. Rev Clin Esp 1989; 184: 238-44.

14. Pileri S, Bocchia M, Baroni CD, Martelli M, Falini B, Sabattini E, et al. Anaplastic large cell lymphoma ( CD30 (+)/Ki-1 (+) ): results of a prospective clinico-pathological study of 69 cases. Br J Haematol 1994; 86: 513-23.

15. Penny RJ, Blaustein JC, Longtine JA. Ki-l positive large cell lymphomas, a heterogeneous group of neoplasms. Cancer 1991; 68: 362-73.

16. Cabanillas F, Romaguerra J, Manning J. Ki-1 positive large cell lym- 
phoma: is it a clinical entity?. Fifth International Conference on Malignant Lymphomas. Lugano 1993; June 9-12. Abstract book 10.

17. Falini B, Pileri S, Martelli MF. Histological and inmunohistological analysis of human lymphomas. Critical Rev Oncol / Haematol 1989; 9: 351-19.

18. Radaszkiewicz T, Simonitsch I, Mosberger I, Chott A. Anaplastic large cell lymphoma: morphology and clinicopathologic correlations. Fifth International Conference on Malignant Lymphomas. Lugano 1993; June 9-12 Abstract book 9 .

19. Stein H, Herbst H, Anagnostopoulos I, Niedobitek G, Dallenbach F, Kratzsch HC. The nature of Hodgkin and Reed-Sternberg cells, their association with EBV, and their relationship to anaplastic large cell lymphoma. Ann Oncol 1991; 2: 33-38.

20. Nakamura S, Takagi N, Kojima M, Motoori T, Kitoh K, Osada H, et al. Clinicopathologic study of large cell anaplastic lymphoma (Ki-1 positive large cell lymphoma) among the japanese. Cancer 1991; 68: 118-129.

21. Close PM, Macrae MB, Hammond JM, Aronson I, Johnson CA, Potgieter $\mathrm{PD}$, et al. Anaplastic large cell Ki-1 lymphoma: pulmonary presentation mimicking miliary tuberculosis. Am J Clin Pathol 1993; 99 : 631-6.

22. Chan JKC, Hui PK, Leungs TW, Lau WH, McGuire LJ. Anaplastic large cell Ki-l lymphoma: delineation of two morphological types. Histopathol 1989; 15: 11-34.

23. Chott A, Kaserer K, Augustin I, Vesely M, Heinz R, Oehlinger W, et al. Ki-1 positive large cell lymphoma: a clinicopathologic study of 41 cases. Am J Surg Pathol 1990; 14: 439-48.

24. Greer JP, Kinney MC, Collins RD, Salhani KE, Wolff SN, Hainsworth JD et al. Clinical features of 31 patients with Ki-1 anaplastic large cell lymphoma. J Clin Oncol 1991; 9: 539-47.

25. Stein H, Gerdes J, Tippelmann G, Dienemann D, Schwarting R, O'Connor N, et al. Ki-l lymphoma: experimental and clinical findings. Third International Conference on Malignant Lymphomas. Lugano 1987; June 10-13. Abstract book

26. Shulman LN, Frisard B, Antin JH, Wheeler C, Pinkus G, Magauran N et al. Primary Ki-1 anaplastic large cell lymphoma in adults: clinical characteristics and therapeutic outcome. J Clin Oncol 1993; 11: 937-42.

27. Mason DY, Bastard C, Rimokh R. CD30-positive large cell lymphomas are associated with a chromosomal translocation involving $5 \mathrm{p} 35$. Br J Haematol 1990; 74: 161-8.

28. LeBeau M, Bitter MA, Larson RA. The $\mathrm{t}(2 ; 5)$ (p23;q35): a recurring chromosomal abnormality in Ki-1 positive anaplastic large cell lymphoma. Leuk 1989; 3: 866-70.

29. Rimokh R, Magaud JP, Berger F. A translocation involving a specific breakpoint (q35) on chromosome 5 is characteristic of anaplastic large cell lymphoma (Ki-1 lymphoma). Br J Haematol 1989; 71: 31-6.

30. Stansfeld AG, Diebold J, Kapanci Y, Kelenyi G, Lannert K, Mioduszewska O, et al. Updated Kiel classification for lymphomas. Lancet 1988; 1: 292-293, 603 .

31. Harris NL, Jaffe ES, Stein H, Banks PM, Chan JKC, Clearly ML, et al. A revised European-American classification of lymphoid neoplasms: a proposal from the International Lymphoma Study Group. Blood 1994; 84: 1361-92.

32. Fasola G, Fanin R, Gherlinzoni F, Galieni P, Taruscio D, Frezza G, et al. Serum LDH concentration in non-Hodgkin's lymphomas. Acta Haematol 1984; 72: 231-8.

33. Gandarillas MA, Conde E, Mazorra F, Cuadrado MA, Baro J, Garijo J, et al. Investigación de factores pronósticos en linfomas de células gran- des. Med Clin 1995; 104: 601-7.

34. Li G, Hansmann ML, Zwingers T, Lennert K. Primary lymphomas of the lung: morphological, inmunohistochemical and clinical features. Histopathol 1990; 16: 519-31.

35. Koss MN, Hochholzer L, Nichols PW, Wehunt WD, Lazarus AA. Primary non-Hodgkin's lymphoma and pseudolymphoma of lung: a study of 161 patients. Hum Pathol 1983; 14: 1024-38.

36. Lewis ER, Caskey CI, Fishman ER. Lymphoma of the lung: CT findings in 31 patients. A J R 1991; 156: 711-4.

37. Peterson H, Snider HL, Yam LT, Bowlds CF, Arnn EH, Li CY. Primary pulmonary lymphoma: a clinical and inmunohistochemical study of six cases. Cancer 1985; 56: 805-13.

38. Pileri S, Falini B, Delsol G, Stein H, Baglioni P, Poggi S, et al. Lymphohistiocytic T-cell lymphoma (anaplastic large cell lymphoma CD30 $(+) / \mathrm{Ki}-1$ (+) with a high content of reactive histiocytes). Histopathol 1990; 16: 383-91.

39. Herbst H, Tippelmann G, Anagnostopulos I, Gerdes J, Schwarting R, Boehm $\mathrm{T}$, et al. Inmunoglobulin and $\mathrm{T}$-cell receptor gene rearrangements in Hodgkin's disease and Ki-l positive anaplastic large cell lymphoma: dissociation between phenotype and genotype. Leuk Res 1989; 13 (2): 103-16.

40. Falini B, Pileri S, Stein H, Dienemann D, Dallenbach F, Delsol G, et al Variable expression of leukocyte common antígen (CD45) in CD30 (Ki-1)-positive anaplastic large cell lymphoma: implication for the differential diagnosis between lymphoid and non-lymphoid malignancies. Human Pathol 1990; 21: 624-9.

41. Rosso R, Paulli M, Magrini U, Kindl S, Boveri E, Volpato G, et al. Anaplastic large cell lymphoma, CD30/Ki-1 positive, expressing the CD15/Leu-Mi antigen. Virchow's Arch (B) 1989; 416: 229-35.

42. Gustmann C, Altmannsberger M, Osborn M, Griesser H, Feller AC Cytokeratin expression and vimentin content in large cell anaplastic lymphomas and other non-Hodgkin's lymphomas. Am J Pathol 1991; 138: 1413-22.

43. Leoncini L, Del Vecchio MT, Kraft R, Megha T, Barbini P, Cevenini $\mathrm{G}$, et al. Hodgkin's disease and CD30-positive anaplastic large cell lymphomas: a continuous spectrum of malignant disorders. Am J Clin Pathol 1990; 137: 1047-57.

44. Fisher RI, Gaynor ER, Dahlberg S, Oken MM, Grogan TM, Mize EM, et al. Comparison of a standard regimen (CHOP) with three intensive chemotherapy regimens for advanced non-Hodgkin's lymphoma. $\mathrm{N}$ Engl J Med 1993; 328: 1002-6.

45. Falini B, Bolognesi A, Flenghi L, Tazzari PL, Broe MK, Stein H, et al Response of refractory Hodgkin's disease to monoclonal anti-CD30 immunotoxin. Lancet 1992; 339: 1195-6.

46. Tazzari PL, Bolognesi A, De Totero D, Falini B, Lemoli R, Soria M, et al. Ber-H2 (anti-CD30)-saporin inmunotoxin: a new tool for the treatment of Hodgkin's disease and CD30 (+) lymphoma: in vitro evaluation. Br J Haematol 1992; 81: 203-211.

47. Berkman N, Breuer R, Kramer MR, Polliack A. Pulmonary involvement in lymphoma. Leuk Lymphoma 1996; 20: 229-37.

48. Nicholson AG, Wotherspoon AC, Jones AL, Sheppard MN, Isaacson PG, Corrin B. Pulmonary B-cell non-Hodgkin's lymphoma associated with autoimmune disorders: a clinicopathological review of six cases. Eur Respir J 1996; 9: 2022-5.

49. Lee CK, Levitt SH. Long-term follow-up of pathologic stage I large cell non-Hodgkin's lymphoma patients after primary radiotherapy. Am J Clin Oncol 1996; 19: 93-8. 